\title{
Checking risky events is enough for local policies
}

\author{
Massimo Bartoletti, Pierpaolo Degano, Gian Luigi Ferrari \\ Dipartimento di Informatica, Università di Pisa, Italy \\ \{bartolet, degano, giangi\}@di.unipi.it
}

\begin{abstract}
An extension of the $\lambda$-calculus is proposed to study historybased access control. It allows for parametrized security policies with a possibly nested, local scope. To govern the rich interplay between local policies, we propose a combination of static analysis and dynamic checking. A type and effect system extracts from programs a correct approximation to the histories obtainable at run-time. A further static analysis over these approximations determines how to instrument code so to enforce the desired security constraints. The execution monitor, based on finite-state automata, runs efficiently the instrumented code.
\end{abstract}

\section{Introduction}

Access control is crucial for securing the execution of mobile applications. Access control policies specify which operations can be executed by possibly untrusted components on sensible resources such as files, communication channels, and so on. Indeed, an attacker may gain full control over a system by getting improper access to critical resources.

Current software technologies enforce access control policies by exploiting different mechanisms. In the Java Virtual Machine, as well as in the .Net Common Language Runtime, stack inspection computes the run-time access rights of code by examining the stack of method invocations. Code includes special time-consuming instructions, called local security checks, that guard access to critical resources. Methods are associated with a static set of permissions, to reflect the trustedness of code. At run-time, a resource access is granted whenever all methods in the call stack have the required permission.

Stack inspection and local security checks offer a pragmatic setting for access control, with a strong bias towards implementation. However, they suffer from two main shortcomings. First, it is difficult to place the needed checks at the relevant points in the code, and even more difficult is guaranteeing that they suffice for enforcing the intended security policy. Second, stack inspection may fail to enforce some security constraints, because it relies on the call stack only. Indeed, the access rights of a certain method are no longer affected by the execution of an untrusted one, after it has been popped from the call stack. This may be harmful, e.g. when trusted code depends on the results supplied by untrusted code [12]. In other words, stack inspection and local security checks are not an appropriate abstraction for security. 
Some alternatives to stack inspection have been explored. In history-based access control, the actual access rights of a piece of code depend on (a suitable abstraction of) the whole execution. This approach has been receiving major attention, at both levels of foundations $[2,4,11,19]$ and of language design and implementation $[1,9]$. Another paradigm proposes to encapsulate code within wrappers that enforce local security policies [18]. A wrapper monitors the execution and aborts it when about to violate some active local policy.

We have recently proposed $\lambda^{[]}$, an extension of the $\lambda$-calculus that reconciles history-based access control with local policies [4]. Local security properties are regular properties of histories and have a possibly nested, local scope. A policy framing $\varphi[e]$ indicates that the program $e$ is protected by the local policy $\varphi$, i.e. the history must always respect $\varphi$ while evaluating $e$.

In [4] we have verified local security policies through a static analysis, based on a type and effect system [20]. The effect approximates the run-time behaviour of a program. Model checking the effect ensures that there will be no security violations at run-time. The static approach avoids the need for an execution monitor to enforce local policies. Also, it avoids keeping track of the whole execution history, which may grow unbound.

Because of the static approximation, some programs may be discarded even though they would be secure, possibly just by adding a limited amount of runtime checking. We propose here a mixed approach to access control, that efficiently combines static analysis and run-time checking. Our technique discards no programs while keeping security. Intuitively, we compile a program with policy framings into an equivalent one without framings, but instrumented with local checks. Our static analysis determines which checks are needed and where they must be inserted to obtain a program respecting the given security requirements. The execution monitor is essentially a finite-state automaton associated with the relevant security policies.

Technically, we define a novel type and effect system; the types are mostly standard and the effects are history grammars. These are context-free grammars representing all possible execution histories, including the scopes of the local policies. A further static analysis over history grammars, called risky events analysis, determines the program points where security violations may occur. The risky events analysis is then exploited to generate the set of checks needed to safely instrument the original code at the right points. The original and instrumented programs indeed satisfy the same security constraints. It is worthwhile noting that the execution monitor controlling the instrumented program only needs a

finite amount of information. More precisely, it suffices to record the states of the finite-state automata associated with checks on risky events.

\section{A motivating example}

To illustrate our approach, consider a simple web browser that runs applets. Each applet must obey a general usage policy $\varphi$ : only open files can be read. Moreover, a user can supply the browser with his own security policy, to be 
enforced on all applet executions. In this example, the user policy says that, after having read a local file, an applet can no longer connect to the network.

The browser is a function that processes the applet $x$ and the user policy $\varphi^{\prime}$. Since policies are not first class expressions in our calculus, the parameter $\varphi^{\prime}$ is rendered as a closure $p=\lambda y \cdot \varphi^{\prime}[y *]$, where $*$ stands for the unit value.

$$
\text { Browser }=\lambda x \cdot \lambda p \cdot \varphi[p x] ; \text { Browser }
$$

We consider a simple editor applet, that accesses a local file $y$ and may save it on a given site, identified by a URL $u$ :

$$
\text { Editor }=\lambda y . \lambda u . \operatorname{open}(y) ; \operatorname{read}(y) ; * ;(\text { if } b \text { then } \operatorname{connect}(u)) ; * ; \operatorname{close}(y)
$$

Note that our editor is overly simplified, because we are only interested in the security-relevant events it can generate; the unit value $*$ indeed represents the sequences of operations that do not affect security.

We also have an attacker applet, that tries to spoof the browser by executing it with a very liberal user policy $\lambda y . y *$ which imposes no constraints. The attacker attempts to force the editor reading the secret password file pwd and then saving it on the attacker site evil.org.

$$
\text { Attacker }=\lambda w . \text { Browser }(\lambda z . \text { Editor pwd evil.org })(\lambda y . y *)
$$

The following trace illustrates the behaviour of the attacker applet, sandboxed with the user policy $\varphi^{\prime}$ by the browser. The program states are pairs, whose first component is a history (a sequence of access events $-\varepsilon$ denotes the empty one) and the second component is the program continuation.

$$
\begin{aligned}
& \varepsilon, \text { Browser Attacker }\left(\lambda y . \varphi^{\prime}[y *]\right) \\
\rightarrow & \varepsilon, \varphi\left[\left(\lambda y . \varphi^{\prime}[y *]\right) \text { Attacker }\right] ; \text { Browser } \\
\rightarrow & \varepsilon, \varphi\left[\varphi^{\prime}[\text { Attacker } *]\right] ; \text { Browser } \\
\rightarrow & \varepsilon, \varphi\left[\varphi^{\prime}[\text { Browser }(\lambda z . \text { Editor } \text { pwd evil.org })(\lambda y . y *)]\right] ; \text { Browser } \\
\rightarrow & \varepsilon, \varphi\left[\varphi^{\prime}[\varphi[(\lambda y . y *)(\lambda z . \text { Editor } \text { pwd evil.org })] ; \text { Browser }]\right] ; \text { Browser } \\
\rightarrow & \left.\varepsilon, \varphi\left[\varphi^{\prime}[\varphi[\text { Editor pwd evil.org })] ; \text { Browser }\right]\right] ; \text { Browser } \\
\rightarrow & \varepsilon, \varphi\left[\varphi^{\prime}[\varphi[\text { open }(\mathrm{pwd}) ; \operatorname{read}(\mathrm{pwd}) ; * ; \text { if } b \text { then } \operatorname{connect}(\text { evil.org }) ; \cdots] \cdots\right. \\
\rightarrow & \text { open }(\mathrm{pwd}), \varphi\left[\varphi^{\prime}[\varphi[\operatorname{read}(\mathrm{pwd}) ; * ; \text { if } b \text { then } \operatorname{connect}(\text { evil.org }) ; \cdots] \cdots\right. \\
\rightarrow & \operatorname{open}(\mathrm{pwd}) \operatorname{read}(\mathrm{pwd}), \varphi\left[\varphi^{\prime}[\varphi[\text { if } b \text { then } \operatorname{connect}(\text { evil.org }) ; * ; \operatorname{close}(\mathrm{pwd})] \cdots\right.
\end{aligned}
$$

If $b$ evaluates to true, then a security exception is thrown, because the history:

$$
\text { open (pwd) read(pwd) connect(evil .org) }
$$

would violate the active policy $\varphi^{\prime}$. Note however that the connect event in the editor is the only program point where the policy $\varphi^{\prime}$ is violated. Instead, all possible runs obey the file usage policy $\varphi$. These observations suggest us to implement the access control mechanism by inserting local checks just before 
risky events, i.e. the program points about to violate policies. In our example, the original editor will be transformed into the equivalent:

$$
\begin{aligned}
\text { Editor }^{\prime}=\lambda x . \lambda u . & \text { open }(x) ; \operatorname{read}(x) ; * ; \\
& \left(\text { if } b \text { then check } \varphi^{\prime} \text { in } \operatorname{connect}(u)\right) ; * ; \operatorname{close}(x)
\end{aligned}
$$

Furthermore, all the policy framings are removed, and expressions are instrumented to record the set of active policies. Our goal is finding therefore the risky program points and the involved policies.

\section{The Language $\lambda^{[]}$}

We consider a call-by-value $\lambda$-calculus enriched with access events and local security policies. This language is called $\lambda^{[]}$, and has been first introduced in [4]. We present here an extension of the calculus that features parametrized access events, and a new type and effect system that is suitable for instrumentation with local checks. An access event $\alpha^{\ell}(c)$ abstracts from a security-relevant operation. The symbol $\alpha \in$ Act stands for an action (e.g. reading a file), while the parameter $c \in \operatorname{Res}$ is the resource upon which the action is taken (e.g. a file name). The label $\ell \in$ Lab uniquely identifies an access event in an expression. We assume the sets Act, Res and Lab to be pairwise disjoint.

Sequences $\eta$ of access events are called histories. Security policies $\varphi \in \Pi$ are regular properties of histories, universally quantified over resources. An instantiated policy $\varphi(c)$ can be phrased as a regular expression, and enforced by a finite state automaton (see below for details). A policy framing $\varphi[e]$ localizes the scope of the policy $\varphi$ to the expression $e$; framings can be arbitrarily nested. To enhance readability, our calculus comprises conditional expressions and named abstractions $\left(z\right.$ in $e^{\prime}=\lambda_{z} x$.e stands for $e^{\prime}$ itself within $e$ ). We omit the definition of guards $b$, as they are not relevant for the subsequent technical development.

\begin{tabular}{|c|c|}
\hline $\begin{aligned} e, e^{\prime}::= & x \\
& c \\
& \alpha^{\ell}(r) \\
& \text { if } b \text { then } e \text { else } e^{\prime} \\
& \lambda_{z} x . e \\
& e e^{\prime} \\
& \varphi[e]\end{aligned}$ & $\begin{array}{l}\text { variable } \\
\text { constant } \\
\text { access event } \\
\text { conditional } \\
\text { abstraction } \\
\text { application } \\
\text { policy framing }\end{array}$ \\
\hline
\end{tabular}

$\lambda^{[]}$expressions

Variables, constants, abstractions, and failures are the values $v$ of $\lambda^{[]}$. A failure fail $_{\ell, \varphi(c)}$ represents a computation that is about to violate the policy $\varphi(c)$ by generating an event $\alpha^{\ell}\left(c^{\prime}\right)$. We assume that, for any expression $e$, label $\ell$ and policy $\varphi(c)$, e fail $l_{\ell, \varphi(c)}=$ fail $_{\ell, \varphi(c)} e=\varphi^{\prime}\left[\right.$ fail $\left._{\ell, \varphi(c)}\right]=$ fail $_{\ell, \varphi(c)}$. We write $*$ for a fixed, closed, event-free, non-failure value, and $\lambda . e$ for $\lambda x . e$, for $x \notin f v(e)$. The following abbreviation is standard: $e ; e^{\prime}=\left(\lambda . e^{\prime}\right) e$. Also, we write $\alpha$ instead of 
$\alpha(c)$ when the resource parameter $c$ is immaterial. Without loss of generality, we assume that each framing has an opening event, i.e. for all $\varphi[e]$, the expression $e$ is of the form $\alpha(c) ; e^{\prime}$, for some $\alpha, c$ and $e^{\prime}$. This opening event can be a dummy event with no influence on security. Note that we also allow for parametrized events $\alpha^{\ell}(r)$, where $r$ ranges over resources and variables.

We define the behaviour of $\lambda^{[]}$expressions through the following small-step operational semantics. The configurations are pairs $\eta, e$, where $e$ may denote both expressions and failures. A transition $\eta, e \rightarrow \eta^{\prime}, e^{\prime}$ means that, starting from a history $\eta$, the expression $e$ may evolve to $e^{\prime}$, possibly extending $\eta$ to $\eta^{\prime}$. We write $\eta \models \varphi(c)$ when the history $\eta$ satisfies the policy $\varphi(c)$. We assume as given a total function $\mathcal{B}$ that evaluates the guards in conditionals, and a function $\operatorname{lab}(\eta)$ that returns the last label $\ell_{k}$ from a history $\eta=\alpha_{1}^{\ell_{1}}\left(c_{1}\right) \cdots \alpha_{k}^{\ell_{k}}\left(c_{k}\right)$. Notice that there is no need to define $\ell(\varepsilon)$, because of the assumed opening event.

\section{Operational semantics of $\lambda^{[]}$}

$$
\begin{gathered}
\frac{\eta, e_{1} \rightarrow \eta^{\prime}, e_{1}^{\prime}}{\eta, e_{1} e_{2} \rightarrow \eta^{\prime}, e_{1}^{\prime} e_{2}} \quad \frac{\eta, e_{2} \rightarrow \eta^{\prime}, e_{2}^{\prime}}{\eta, v e_{2} \rightarrow \eta^{\prime}, v e_{2}^{\prime}} \quad \eta,\left(\lambda_{z} x . e\right) v \rightarrow \eta, e\left\{v / x, \lambda_{z} x . e / z\right\} \\
\eta, \alpha^{\ell}(c) \rightarrow \eta \alpha^{\ell}(c), * \quad \eta, \text { if } b \text { then } e_{0} \text { else } e_{1} \rightarrow \eta, e_{\mathcal{B}(b)} \\
\frac{\eta, e \rightarrow \eta^{\prime}, e^{\prime} \quad \forall c . \eta^{\prime} \models \varphi(c)}{\eta, \varphi[e] \rightarrow \eta^{\prime}, \varphi\left[e^{\prime}\right]} \quad \frac{\eta, e \rightarrow \eta^{\prime}, e^{\prime} \quad \exists c . \eta^{\prime} \not \models \varphi(c)}{\eta, \varphi[e] \rightarrow \eta, f a i l_{l a b\left(\eta^{\prime}\right), \varphi(c)}} \quad \eta, \varphi[v] \rightarrow \eta, v
\end{gathered}
$$

The rules above are mostly standard, except for those governing evaluation within policy framings. An expression $\varphi[e]$ can evolve to $\varphi\left[e^{\prime}\right]$, provided that the resulting history $\eta^{\prime}$ satisfies all the possible instantiations $\varphi(c)$; a failure occurs when $\varphi(c)$ is violated for some $c$. Eventually, values leave the scope of policies. More concrete operational semantics could reduce the number of instantiated policies, e.g. by looking only at the constants occurring in $\eta^{\prime}$, or by selecting constants with a suitable type, like file, memory region, etc.

A security policy $\varphi(c)$ is specified by a deterministic finite-state automaton $A_{\varphi(c)}=\left(\Sigma, Q, q_{0}, \delta\right)$, where $\Sigma=$ Act $\times$ Res is the input alphabet, $Q$ is the set of states, $q_{0} \in Q$ is the start state, and $\delta: Q \times \Sigma \rightarrow Q$ is the transition function. Without loss of generality, we assume a distinguished state $q_{s} \in Q$ which is the only non-accepting state and is a sink, i.e. $\delta\left(q_{s}, \beta\right)=q_{s}$ for any $\beta \in \Sigma$. Note that $\eta \models \varphi(c)$ actually means that $\delta\left(q_{0}, \eta\right) \neq q_{s}$ in the automaton $A_{\varphi(c)}$.

Example 1. To keep small the size of our example, it is convenient to slightly simplify the browser/applet system considered in Section 2.

$$
\begin{aligned}
B & =\lambda_{z} x . \varphi^{\prime}[x *] ; z x \\
A & =\lambda . \text { if } b \text { then } \alpha_{\text {read }}(c) \text { else } \alpha_{\text {connect }} \\
e & =\varphi\left[\alpha_{\text {open }}(c) ; B A ; \alpha_{\text {close }}(c)\right]
\end{aligned}
$$


Let $\ell$ be the label of the event $\alpha_{\text {connect }}$, and assume first that $b$ is true. The evaluation of $e$ goes as follows (for readability, the event labels are omitted):

$$
\begin{aligned}
\varepsilon, e & \rightarrow \alpha_{\text {open }}(c), \varphi\left[B A ; \alpha_{\text {close }}(c)\right] \\
& \rightarrow \alpha_{\text {open }}(c), \varphi\left[\varphi^{\prime}[A *] ; B A ; \alpha_{\text {close }}(c)\right] \\
& \rightarrow \alpha_{\text {open }}(c), \varphi\left[\varphi^{\prime}\left[\text { if } b \text { then } \alpha_{\text {read }}(c) \text { else } \alpha_{\text {connect }}\right] ; B A ; \alpha_{\text {close }}(c)\right] \\
& \rightarrow \alpha_{\text {open }}(c), \varphi\left[\varphi^{\prime}\left[\alpha_{\text {read }}(c)\right] ; B A ; \alpha_{\text {close }}(c)\right] \\
& \rightarrow \alpha_{\text {open }}(c) \alpha_{\text {read }}(c), \varphi\left[\varphi^{\prime}[*] ; B A ; \alpha_{\text {close }}(c)\right] \\
& \rightarrow \alpha_{\text {open }}(c) \alpha_{\text {read }}(c), \varphi\left[* ; B A ; \alpha_{\text {close }}(c)\right]
\end{aligned}
$$

Assume now that $b$ becomes false. Then, the computation proceeds as follows:

$$
\begin{aligned}
\cdots & \rightarrow \alpha_{\text {open }}(c) \alpha_{\text {read }}(c), \varphi\left[B A ; \alpha_{\text {close }}(c)\right] \\
& \rightarrow \alpha_{\text {open }}(c) \alpha_{\text {read }}(c), \varphi\left[\varphi^{\prime}[A *] ; B A ; \alpha_{\text {close }}(c)\right] \\
& \rightarrow \alpha_{\text {open }}(c) \alpha_{\text {read }}(c), \varphi\left[\varphi^{\prime}\left[\text { if } b \text { then } \alpha_{\text {read }}(c) \text { else } \alpha_{\text {connect }}\right] ; B A ; \alpha_{\text {close }}(c)\right] \\
& \rightarrow \alpha_{\text {open }}(c) \alpha_{\text {read }}(c), \varphi\left[\varphi^{\prime}\left[\alpha_{\text {connect }}\right] ; B A ; \alpha_{\text {close }}(c)\right] \\
& \rightarrow \text { fail }_{\ell, \varphi^{\prime}(c)}
\end{aligned}
$$

The computation fails, because generating the event $\alpha_{\text {connect }}$ would make the history $\alpha_{\text {open }}(c) \alpha_{\text {read }}(c) \alpha_{\text {connect }}$ violate the policy $\varphi^{\prime}(c)$.

Figure 1 displays the universally quantified automata for the usage policy $\varphi$ and the user policy $\varphi^{\prime}$ introduced in Section 2. We only draw the arcs labelled with actions relevant to the policy in hand, while the other actions are intended to originate self-loops, omitted in the figure. The actual security automata are obtained by instantiating the parameter $x$ to a resource $c$. The sink state $q_{s}$ is shared by both automata, while $q_{0}$ and $q_{0}^{\prime}$ are the start states.
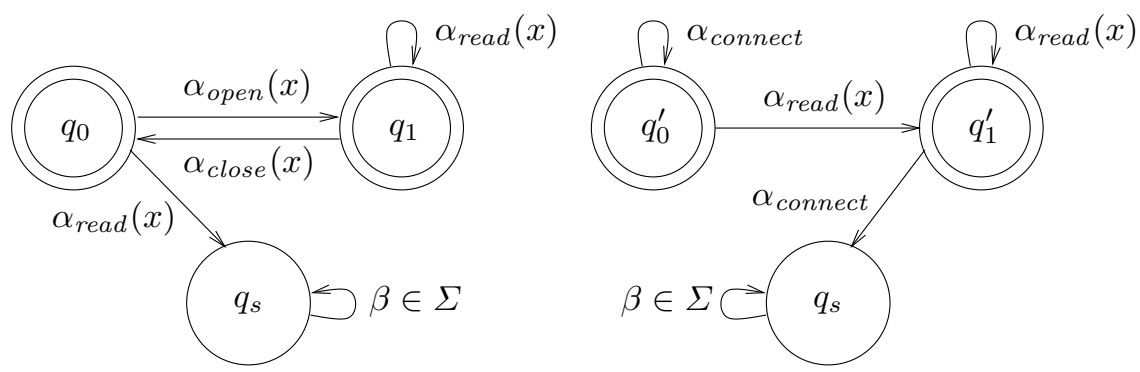

Fig. 1. Security automata $A_{\varphi(x)}$ (left) and $A_{\varphi^{\prime}(x)}$ (right). 


\section{Extracting History Grammars}

We now introduce a type and effect system for $\lambda^{[]}$, that we shall use for approximating the aspects of the expression behaviour relevant to security.

Given an expression $e$, it is convenient to label each of its subexpressions. For simplicity, we shall use injective labellings only, and we coherently extend the labelling of an event $\alpha^{\ell}(r)$ to $\alpha^{\ell}\left(r^{\ell^{\prime}}\right)$. To keep track of the framings, we shall use the special framing events $[\varphi \text { and }]_{\varphi}$ that stand respectively for opening and closing the scope of the policy $\varphi$. We denote with Frm the set $\left\{[\varphi,]_{\varphi} \mid \varphi \in \Pi\right\}$.

Types $\tau$ and type environments $\Gamma$ are defined as follows, where $R \subseteq$ Res.

Types and Type Environments

$$
\tau::=\text { unit }|R| \tau \stackrel{H}{\longrightarrow} \tau \quad \Gamma::=\emptyset \mid \Gamma ; x: \tau \quad(x \notin \operatorname{dom}(\Gamma))
$$

The types are much the same of the implicitly-typed $\lambda$-calculus (the set type $R$ is used for resources), while the effects are our history grammars. These are context-free grammars $H=(T, N, \ell, P)$, where $T=$ Act $\cup \operatorname{Res} \cup$ Frm is the set of terminal symbols, $N=$ Lab is the set of non-terminals, $\ell \in N$ is the start symbol, and $P \subseteq N \times(T \cup N)^{*}$ is the set of productions. We shall use the following standard notation: $(\ell, P)$ for $H, \Rightarrow$ for the derivation relation, $\mathcal{L}(H)$ for the language generated by $H$, and $\varepsilon$ for the empty string.

The effect $H$ of an expression $e$ represents all the possible run-time histories of $e$, and also records entering and exiting from the scope of security policies.

Example 2. Consider the following (labelled) expression:

$$
e^{0}=\alpha^{1}\left(c^{2}\right) ; \varphi\left[\left(\text { if } b \text { then } \alpha^{\prime 5}\left(c^{\prime 6}\right) \text { else } *^{7}\right)^{4}\right]^{3}
$$

The history grammar extracted from $e$ is as follows (the underlined label stands for the start symbol).

$$
\begin{array}{ll}
\underline{0} \rightarrow 13 & 4 \rightarrow 5 \mid 7 \\
1 \rightarrow \alpha c & 5 \rightarrow \alpha^{\prime} c^{\prime} \\
2 \rightarrow \varepsilon & 6 \rightarrow \varepsilon \\
3 \rightarrow\left[{ }_{\varphi} 4\right]_{\varphi} & 7 \rightarrow \varepsilon
\end{array}
$$

We compare below a computation of $e$ (left) with a derivation of $H$ (right), assuming $b$ true and $\varphi$ always respected.

$$
\begin{aligned}
\varepsilon, e & \rightarrow \alpha(c), \varphi\left[\text { if } b \text { then } \alpha^{\prime}\left(c^{\prime}\right) \text { else } *\right] & \underline{0} & \Rightarrow 13 \Rightarrow \alpha c 3 \\
& \rightarrow \alpha(c), \varphi\left[\alpha^{\prime}\left(c^{\prime}\right)\right] & & \Rightarrow \alpha c\left[{ }_{\varphi} 4\right]_{\varphi} \Rightarrow \alpha c\left[{ }_{\varphi} 5\right]_{\varphi} \\
& \rightarrow \alpha(c) \alpha^{\prime}\left(c^{\prime}\right), \varphi[*] & & \Rightarrow \alpha c\left[{ }_{\varphi} \alpha^{\prime} c^{\prime}\right]_{\varphi}
\end{aligned}
$$

The framing events in $\alpha c\left[{ }_{\varphi} \alpha^{\prime} c\right]_{\varphi}$ mean that the policy $\varphi$ is active when the event $\alpha^{\prime}\left(c^{\prime}\right)$ is generated, so the history $\alpha(c) \alpha^{\prime}\left(c^{\prime}\right)$ must obey $\varphi$, otherwise the computation fails. 
A typing judgment $\Gamma, H \vdash e: \tau$ means that the expression $e$ evaluates to a value of type $\tau$, and produces a history represented by the effect $H$. The history grammar $H$ in the functional type $\tau \stackrel{H}{\longrightarrow} \tau^{\prime}$ describes the latent effect associated with an abstraction, i.e. one of the histories in $\mathcal{L}(H)$ is generated when applying that abstraction to a value. The relation $\Gamma, H \vdash e: \tau$ is the least one closed under the following rules. For brevity, we shall omit immaterial labels, and we shall write $\underline{\ell} \rightarrow \gamma, P$ for $(\ell,\{\ell \rightarrow \gamma\} \cup P)$, and $\ell \rightarrow \gamma \mid \gamma^{\prime}$ when $\ell \rightarrow \gamma$ and $\ell \rightarrow \gamma^{\prime}$.

Type and Effect System for $\lambda^{[]}$

$$
\begin{gathered}
\frac{R \subseteq \operatorname{Res}}{\Gamma, \underline{\ell} \rightarrow \varepsilon \vdash c^{\ell}:\{c\} \cup R} \quad \frac{\Gamma,\left(\ell^{\prime}, P\right) \vdash r: R}{\Gamma,\{\underline{\ell} \rightarrow \alpha c: c \in R\}, P \vdash \alpha^{\ell}\left(r^{\ell^{\prime}}\right): \text { unit }} \\
\frac{\Gamma ; x: \tau ; z: \tau \stackrel{\left(\ell^{\prime}, P\right)}{\longrightarrow} \tau^{\prime},\left(\ell^{\prime}, P\right) \vdash e: \tau^{\prime}}{\Gamma, \underline{\ell} \rightarrow \varepsilon \vdash\left(\lambda_{z} x \cdot e\right)^{\ell}: \tau \stackrel{\left(\ell^{\prime}, P\right)}{\longrightarrow} \tau^{\prime}} \\
\frac{\Gamma, \underline{\ell} x^{\ell}: \Gamma(x)}{\Gamma, \underline{\ell} \rightarrow \varepsilon \vdash *^{\ell}: \text { unit }} \quad \frac{\Gamma,\left(\ell_{0}, P_{0}\right) \vdash e_{0}: \tau \stackrel{\left(\ell_{2}, P_{2}\right)}{\longrightarrow} \tau^{\prime} \Gamma,\left(\ell_{1}, P_{1}\right) \vdash e_{1}: \tau}{\Gamma, \underline{\ell} \rightarrow \ell_{0} \ell_{1} \ell_{2}, P_{0} \cup P_{1} \cup P_{2} \vdash\left(e_{0} e_{1}\right)^{\ell}: \tau^{\prime}} \\
\frac{\Gamma,\left(\ell_{0}, P_{0}\right) \vdash e_{0}: \tau \quad \frac{\Gamma,\left(\ell_{1}, P_{1}\right) \vdash e_{1}: \tau}{\Gamma, \underline{\ell} \rightarrow \ell_{0} \mid \ell_{1}, P_{0} \cup P_{1} \vdash\left(\text { if } b \text { then } e_{0} \text { else } e_{1}\right)^{\ell}: \tau}}{\Gamma,\left(\ell^{\prime}, P^{\prime}\right) \vdash e: \tau} \quad \frac{\Gamma, H \vdash e: \tau}{\Gamma, H^{\prime} \vdash e: \tau} \mathcal{L}\left(H^{\prime}\right) \supseteq \mathcal{L}(H)
\end{gathered}
$$

Typing judgments are standard, and allow for weakening of both resource types (in the first rule) and effects (in the last rule). The effects in the rule for application are concatenated according to the evaluation order of the callby-value semantics (function, argument, latent effect). The actual effect of an abstraction is the empty language, while the latent effect is equal to the actual effect of the function body.

Example 3. To clarify the role of weakening, consider the following expressions:

$$
\begin{aligned}
& e_{1}=\left(\left(\lambda x \cdot \alpha^{3}\left(x^{4}\right)\right)^{2}\left(\text { if } b \text { then } c^{6} \text { else } c^{\prime 7}\right)^{5}\right)^{1} \\
& e_{2}=\left(\text { if } b \text { then }\left(\lambda x \cdot \alpha^{13}\left(c^{14}\right)\right)^{12} \text { else }\left(\lambda y \cdot \alpha^{16}\left(c^{17}\right)\right)^{15}\right)^{11}
\end{aligned}
$$

For $e_{1}$, let $\Gamma=\left\{x:\left\{c, c^{\prime}\right\}\right\}$. The typing derivation needs weakening the set types associated with the constants $c$ and $c^{\prime}$. For readability, we often omit labels, the 
environment $\Gamma$, and we only show the last productions added to effects.

$$
\frac{\frac{4 \rightarrow \varepsilon \vdash x:\left\{c, c^{\prime}\right\}}{3 \rightarrow \alpha c \mid \alpha c^{\prime} \vdash \alpha(x): \text { unit }}}{\frac{2 \rightarrow \varepsilon \vdash \lambda x . \alpha(x):\left\{c, c^{\prime}\right\} \stackrel{3}{\rightarrow} \text { unit }}{2 \rightarrow 253 \vdash e_{1}: \text { unit }}} \frac{6 \rightarrow \varepsilon \vdash c:\{c\} \cup\left\{c^{\prime}\right\} \quad 7 \rightarrow \varepsilon \vdash c^{\prime}:\left\{c^{\prime}\right\} \cup\{c\}}{5 \rightarrow 6 \mid 7 \vdash \text { if } b \text { then } c \text { else } c^{\prime}:\left\{c, c^{\prime}\right\}}
$$

For $e_{2}$, let $\Gamma=\{x: \tau, y: \tau\}$. The typing derivation weakens the effects to unify the types in the rule for conditionals (note the introduction of the new label $\ell$ ).

$$
\begin{array}{cc}
\frac{\frac{14 \rightarrow \varepsilon \vdash c:\{c\}}{13 \rightarrow \alpha c \vdash \alpha(c): \text { unit }}}{\frac{\ell \rightarrow 13 \mid 16 \vdash \alpha(c): \text { unit }}{12 \rightarrow \varepsilon \vdash \lambda x . \alpha(c): \tau \stackrel{\ell}{\rightarrow} \text { unit }}} \quad \frac{\frac{17 \rightarrow \varepsilon \vdash c:\{c\}}{16 \rightarrow \alpha^{\prime} c \vdash \alpha^{\prime}(c): \text { unit }}}{11 \rightarrow 13 \mid 16 \vdash \alpha^{\prime}(c): \text { unit }} \\
\hline 12 \mid 15 \vdash e_{2}: \tau \stackrel{\ell}{\rightarrow} \text { unit }
\end{array}
$$

Example 4. Consider the browser and the applet defined in Example 1. After labelling all subexpressions, we obtain:

$$
\begin{aligned}
e^{0} & =\varphi\left[\left(\alpha_{\text {open }}^{2}\left(c^{3}\right) ;\left(B^{10} A^{20}\right)^{4} ; \alpha_{\text {close }}^{5}\left(c^{6}\right)\right)^{1}\right] \\
B^{10} & =\lambda_{z} x \cdot\left(\varphi^{\prime}\left[\left(x^{14} *^{15}\right)^{13}\right]^{12} ;\left(z^{17} x^{18}\right)^{16}\right)^{11} \\
A^{20} & =\lambda y \cdot\left(\operatorname{if} b \text { then } \alpha_{\text {read }}^{22}\left(c^{23}\right) \text { else } \alpha_{\text {connect }}^{24}\right)^{21}
\end{aligned}
$$

Let $\Gamma=\left\{x:\right.$ unit $\stackrel{\ell, P}{\longrightarrow}$ unit, $y: \tau, z: \tau^{\prime} \stackrel{\ell^{\prime}, P^{\prime}}{\longrightarrow}$ unit $\}$, for some $\ell, \ell^{\prime}, P, P^{\prime}, \tau, \tau^{\prime}$. For the expression $A$, we have the following typing derivation:

$$
\begin{aligned}
& \frac{\underline{23} \rightarrow \varepsilon \vdash c:\{c\}}{\underline{22 \rightarrow \alpha_{\text {read }} c \vdash \alpha_{\text {read }}(c): \text { unit }}} \quad \underline{24} \rightarrow \alpha_{\text {connect }} \vdash \alpha_{\text {connect }}: \text { unit } \\
& \underline{21} \rightarrow 22 \mid 24 \vdash \text { if } b \text { then } \alpha_{\text {read }}(c) \text { else } \alpha_{\text {connect }}: \text { unit } \\
& \underline{20} \rightarrow \varepsilon \vdash A: \tau \stackrel{21, P_{A}}{\longrightarrow} \text { unit }
\end{aligned}
$$

where $P_{A}$ collects all the productions generated in the previous deduction:

$$
\begin{array}{rl}
\underline{21} \rightarrow 22 \mid 24 & 23 \rightarrow \varepsilon \\
22 \rightarrow \alpha_{\text {read }} c & 24 \rightarrow \alpha_{\text {connect }}
\end{array}
$$

The following typing derivation is possible for the body of $B$ (we here shortcut typing, by assuming the abbreviation for ; introduced above).

$$
\frac{\frac{\frac{\underline{14} \rightarrow \varepsilon \vdash x: \text { unit } \stackrel{\ell, P}{\longrightarrow} \text { unit } \quad \underline{15} \rightarrow \varepsilon \vdash *: \text { unit }}{\underline{13} \rightarrow 1415 \ell \vdash x *: \text { unit }}}{\underline{12} \rightarrow\left[\varphi^{\prime} 13\right]_{\varphi^{\prime}} \vdash \varphi^{\prime}[x *]: \text { unit }} \quad \frac{\vdots}{\underline{16} \rightarrow 1718 \ell^{\prime} \vdash z x: \text { unit }}}{\underline{11 \rightarrow 1216 \vdash \varphi^{\prime}[x *] ; z x: \text { unit }}}
$$


Let $P_{B}$ be the following set of productions:

$$
\begin{array}{ll}
\underline{11} \rightarrow 1216 & 15 \rightarrow \varepsilon \\
12 \rightarrow\left[\varphi^{\prime} 13\right]_{\varphi^{\prime}} & 16 \rightarrow 1718 \ell^{\prime} \\
13 \rightarrow 1415 \ell & 17 \rightarrow \varepsilon \\
14 \rightarrow \varepsilon & 18 \rightarrow \varepsilon
\end{array}
$$

To apply the rule for abstraction, we equate $\tau^{\prime}$ to unit $\stackrel{\ell, P}{\longrightarrow}$ unit, and unify $\left(\ell^{\prime}, P^{\prime}\right)$ with $\left(11, P_{B}\right)$, obtaining $H_{B}=\left(11, P_{B}\left\{11 / \ell^{\prime}\right\}\right)$. Then, we have:

$$
\frac{\left\{x: \text { unit } \stackrel{\ell, P}{\longrightarrow} \text { unit, } z:(\text { unit } \stackrel{\ell, P}{\longrightarrow} \text { unit }) \stackrel{H_{B}}{\longrightarrow} \text { unit }\right\}, H_{B} \vdash \varphi^{\prime}[x *] ; z x: \text { unit }}{\underline{10} \rightarrow \varepsilon \vdash B:(\text { unit } \stackrel{\ell, P}{\longrightarrow} \text { unit }) \stackrel{H_{B}}{\longrightarrow} \text { unit }}
$$

To apply $B$ to $A$, we solve the constraint unit $\stackrel{\ell, P}{\longrightarrow}$ unit $=\tau \stackrel{21, P_{A}}{\longrightarrow}$ unit. This yields $\tau=$ unit, $\ell=21$, and $P=P_{A}$, so enabling the following judgement:

$$
\frac{B:\left(\text { unit } \stackrel{21, P_{A}}{\longrightarrow} \text { unit }\right) \stackrel{H_{B}\{21 / \ell\}}{\longrightarrow} \text { unit } \quad A: \text { unit } \stackrel{21, P_{A}}{\longrightarrow} \text { unit }}{\underline{4} \rightarrow 102011 \vdash B A: \text { unit }}
$$

We can eventually reconstruct the type and effect of $e$ (notice that we cheat again and solve two sequential compositions in one step, here and in the labelling).

$$
\frac{\frac{\underline{3} \rightarrow \varepsilon \vdash c:\{c\}}{\underline{\underline{2} \rightarrow \alpha_{\text {open }} c \vdash \alpha_{\text {open }}(c): \text { unit }} \quad \frac{\vdots}{\underline{4} \rightarrow 102011 \vdash B A: \text { unit }}} \quad \cdots}{\underline{1} \rightarrow 245 \vdash \alpha_{\text {open }}(c) ;(B A) ; \alpha_{\text {close }}(c): \text { unit }}
$$

Summing up, the history grammar of $e$ is:

$$
\begin{aligned}
& \underline{0} \rightarrow\left[{ }_{\varphi} 1\right]_{\varphi} \\
& 10 \rightarrow \varepsilon \\
& 20 \rightarrow \varepsilon \\
& 1 \rightarrow 245 \\
& 11 \rightarrow 1216 \\
& 21 \rightarrow 22 \mid 24 \\
& 2 \rightarrow \alpha_{\text {open }} c \\
& 12 \rightarrow\left[\varphi^{\prime} 13\right]_{\varphi^{\prime}} \\
& 22 \rightarrow \alpha_{\text {read }} c \\
& 3 \rightarrow \varepsilon \\
& 13 \rightarrow 141521 \\
& 23 \rightarrow \varepsilon \\
& 4 \rightarrow 102011 \\
& 14 \rightarrow \varepsilon \\
& 24 \rightarrow \alpha_{\text {connect }} \\
& 5 \rightarrow \alpha_{\text {close }} c \\
& 15 \rightarrow \varepsilon \\
& 6 \rightarrow \varepsilon \\
& 16 \rightarrow 171811 \\
& 17 \rightarrow \varepsilon \\
& 18 \rightarrow \varepsilon
\end{aligned}
$$

Now it is convenient to introduce the following definition. Given $w \in T^{*}$, let $w^{b}$ be the string obtained from $w$ by pruning all the framing events, and by replacing each substring $\alpha c$ with $\alpha(c)$. Back to Example 2, if $w=\alpha c\left[{ }_{\varphi} \alpha^{\prime} c^{\prime}\right]_{\varphi}$ then $w^{b}=\alpha(c) \alpha^{\prime}\left(c^{\prime}\right)$. The next theorem ensures that our type and effect system indeed approximates the actual run-time histories. 
Theorem 1 (Correctness). Let $\Gamma, H \vdash e: \tau$ and $\varepsilon, e \rightarrow^{*} \eta, e^{\prime}$. Then, there exist $w \in T_{H}^{*}$ and $\gamma \in\left(T_{H} \cup N_{H}\right)^{*}$ such that $H \Rightarrow^{*} w \gamma$, and $w^{b}=\eta$.

\section{The Risky Events Analysis}

In this section we exploit history grammars to determine the program points where security violations may occur. This paves us the way for gaining efficiency by discarding policy framings. Actually, we instrument programs with local checks, guarding risky events with those policies that may be violated. Formally, an event $\alpha^{\ell}(c)$ of an expression $e$ is risky for the policy $\varphi\left(c^{\prime}\right)$ when there exists a computation $\varepsilon, e \rightarrow^{*} \eta, f_{a i l} l_{\ell, \varphi\left(c^{\prime}\right)}$. Below, we define a static analysis that extracts an over-approximations of the risky events of $e$ from the history grammar $H$ of $e$.

Our static analysis takes the form of a transition system, whose configurations are quadruples $\gamma, \sigma, \Phi, \zeta$. The component $\gamma$ is a string derivable from $H, \sigma$ is a mapping from policies to security automata states, $\Phi$ is a sequence of (universally quantified) policies, and $\zeta$ is a mapping from event labels to policies. The start configuration is $\ell, \sigma_{0}, \varepsilon, \zeta_{0}$, where $\ell$ is the start symbol of $H, \sigma_{0}$ maps, for all $\varphi$ and $c^{\prime}$ occurring in $e$, the policy $\varphi\left(c^{\prime}\right)$ to the start state of $A_{\varphi\left(c^{\prime}\right)}$, and $\zeta_{0}$ maps each event label to the empty set. Intuitively, $\sigma$ mimics the evolution of the security automata, $\Phi$ records the active policies, and $\zeta$ accumulates the risky events, i.e. whenever $\alpha^{\ell}(c)$ is risky for $\varphi\left(c^{\prime}\right)$, then eventually $\varphi\left(c^{\prime}\right) \in \zeta(\ell)$.

The transition relation $\rightsquigarrow$ is the least one closed under the following rules.

\section{Risky Events Analysis}

$$
\begin{aligned}
& \left.{ }_{\varphi} \gamma, \sigma, \Phi, \zeta \rightsquigarrow_{H} \gamma, \sigma, \Phi \varphi, \zeta \quad\right]_{\varphi} \gamma, \sigma, \Phi \varphi, \zeta \rightsquigarrow_{H} \gamma, \sigma, \Phi, \zeta \\
& \frac{\ell \rightarrow \alpha c \in H \quad \Phi^{\prime}=\left\{\varphi\left(c^{\prime}\right) \mid \varphi \in \Phi \wedge \delta\left(\sigma\left(\varphi\left(c^{\prime}\right)\right), \alpha(c)\right)=q_{s}\right\}}{\ell, \sigma, \Phi, \zeta \rightsquigarrow H \varepsilon, \delta(\sigma, \alpha(c)), \Phi, \zeta\left\{\ell \mapsto \zeta(\ell) \cup \Phi^{\prime}\right\}} \\
& \frac{\ell \rightarrow \gamma \in H \quad \gamma \neq \alpha c}{\ell, \sigma, \Phi, \zeta{ }_{H} \gamma, \sigma, \Phi, \zeta} \quad \frac{\ell, \sigma, \Phi, \zeta \rightsquigarrow_{H} \gamma^{\prime}, \sigma^{\prime}, \Phi^{\prime}, \zeta^{\prime}}{\ell \gamma, \sigma, \Phi, \zeta \rightsquigarrow_{H} \gamma^{\prime} \gamma, \sigma^{\prime}, \Phi^{\prime}, \zeta^{\prime}}
\end{aligned}
$$

The first axiom appends the policy $\varphi$ in the sequence $\Phi$ upon a framing event $[\varphi$; the second deals with the symmetric case $]_{\varphi}$. The central rule considers an event $\alpha^{\ell}(c)$, and checks whether an automaton $A_{\varphi\left(c^{\prime}\right)}$ enters the (non-accepting) sink state, for some active $\varphi \in \Phi$. In that case, the mapping $\zeta$ records the association of $\ell$ with $\varphi\left(c^{\prime}\right)$, i.e. that $\alpha^{\ell}(c)$ is possibly risky for $\varphi\left(c^{\prime}\right)$. The mapping $\sigma$ is updated to reflect the change of state in the automata upon the event $\alpha(c)$ : the next state of the (deterministic) automaton $A_{\varphi\left(c^{\prime}\right)}$ is the one reachable through $\alpha(c)$. For notational convenience, $\delta$ maps homomorphically on $\sigma$, i.e. on the states of the automata, that are all disjoint except for $q_{s}$. The last two rules simply carry over the above on strings $\gamma$. 
Below we define the set of policies act $(e)$ active in an expression $e$. The intuition is that a policy $\varphi$ is active if reducing the current redex involves checking $\varphi$. Notice that no policy is active for values, because they cannot be further reduced, and for conditionals, because evaluating a guard requires no check.

$$
\begin{aligned}
& \operatorname{act}(\alpha(r))=\emptyset \quad \operatorname{act}(\varphi[e])=\{\varphi\} \cup \operatorname{act}(e) \quad \operatorname{act}\left(\text { if } b \text { then } e \text { else } e^{\prime}\right)=\emptyset \\
& \operatorname{act}\left(v e^{\prime}\right)=\operatorname{act}\left(e^{\prime}\right) \quad \operatorname{act}\left(e e^{\prime}\right)=\operatorname{act}(e)(e \neq v) \quad \operatorname{act}(\lambda x . e)=\emptyset
\end{aligned}
$$

The following theorem establishes the soundness of the risky events analysis, by connecting each computation in $\rightarrow$ with one in $\rightsquigarrow$. In particular, when the first leads to a failing history $\eta$, at least one of the security automata used in the second reaches the sink state, and $\zeta$ associates the violated policy with the offending event. In any case, the active policies in the configurations of $\rightarrow$ are precisely recorded by the component $\Phi$ in the configurations of $\rightsquigarrow$.

Theorem 2. Let $\Gamma, H \vdash e_{0}^{\ell}: \tau$, and $\varepsilon, e_{0}^{\ell} \rightarrow^{n} \eta_{n}, e_{n}$. Then, there exist $\gamma, \sigma, \Phi, \zeta$ such that $\ell, \sigma_{0}, \varepsilon, \zeta_{0}{ }_{H}^{*} \gamma, \sigma, \Phi, \zeta$, and:

(2a) if $e_{n}=$ fail $_{\ell^{\prime}, \varphi(c)}$ then $\varphi(c) \in \zeta\left(\ell^{\prime}\right)$ and $\delta(\sigma(\varphi(c)), \eta)=q_{s}$

(2b) otherwise, $\sigma=\delta\left(\sigma_{0}, \eta\right)$, and $\{\varphi \mid \varphi$ occurs in $\Phi\}=\operatorname{act}\left(e_{n-1}\right)$.

We now show that the an over-approximation to the risky events of a program can be computed in a finite amount of time. More precisely, there exists a bound on the length of the computations needed to stabilise $\zeta$.

Theorem 3. Let $\Gamma, H \vdash e: \tau$, for e closed. For each $n \geq 0$, define:

$$
Z_{n}=\left\{\zeta \mid \ell, \sigma_{0}, \varepsilon, \zeta_{0} \rightsquigarrow \stackrel{n}{H} \gamma, \sigma, \Phi, \zeta\right\}
$$

Then, there exists $k$ such that, for all $i \geq 0, Z_{k+i}=Z_{k}$.

Given the set $Z_{k}$ of the above theorem, we hereafter denote with RE the mapping such that $\ell \mapsto\left\{\varphi(c) \in \zeta(\ell) \in Z_{k}\right\}$. Call $\ell H$-risky for $\varphi(c)$ whenever $\varphi(c) \in \operatorname{RE}(\ell)$. Theorems 1,2 and 3 allow us to establish the correctness of the risky events analysis: if $\alpha^{\ell}\left(c^{\prime}\right)$ is risky for $\varphi(c)$, then $\ell$ is $H$-risky for $\varphi(c)$.

Example 5. Consider the browser/applet system analysed in Example 4. The following computation discovers that 24 is a risky event for $\varphi^{\prime}(c)$.

$$
\begin{aligned}
0, \sigma_{0}, \varepsilon, \zeta_{0} & \left.\left.{ }^{*} 22\right]_{\varphi^{\prime}} 165\right]_{\varphi}, \sigma_{1}=\delta\left(\sigma_{0}, \alpha_{\text {open }}(c)\right), \varphi \varphi^{\prime}, \zeta_{0} \\
& \left.\rightsquigarrow 24]_{\varphi^{\prime}} 165\right]_{\varphi}, \sigma_{2}=\delta\left(\sigma_{1}, \alpha_{\text {read }}(c)\right), \varphi \varphi^{\prime}, \zeta_{0} \\
& \left.\rightsquigarrow]_{\varphi^{\prime}} 165\right]_{\varphi}, \delta\left(\sigma_{2}, \alpha_{\text {connect }}\right), \varphi \varphi^{\prime}, \zeta_{0}\left\{24 \mapsto \varphi^{\prime}(c)\right\}
\end{aligned}
$$

\section{Instrumentation with Local Checks}

We eventually exploit the risky events analysis to obtain an expression with local checks only, equivalent to a given expression with local policies. The syntax and operational semantics of the target language $\lambda^{\text {check }}$ follow. 
The target language $\lambda^{\text {check }}$

\begin{tabular}{|c|c|}
\hline $\begin{aligned} e, e^{\prime}::= & x \\
& c \\
& \operatorname{check} \Phi \text { in } \alpha(r) \\
& \text { if } b \text { then } e \text { else } e^{\prime} \\
& \lambda_{z} x . e \\
& e e^{\prime} \\
& \text { enter } \varphi \text { in } e\end{aligned}$ & $\begin{array}{l}\text { variable } \\
\text { constant } \\
\text { guarded event } \\
\text { conditional } \\
\text { abstraction } \\
\text { application } \\
\text { activate policy }\end{array}$ \\
\hline
\end{tabular}

The configurations of the operational semantics are pairs $\sigma, e$. A transition $\Phi \vdash \sigma, e \rightarrow \sigma^{\prime}, e^{\prime}$ means that the expression $e$ reduces to $e^{\prime}$ and the state $\sigma$ of the security automata evolves to $\sigma^{\prime}$, provided that $\Phi$ is the set of active policies. Note that it suffices to record in $\sigma$ the states of those automata $A_{\varphi(c)}$ such that $\varphi(c) \in \operatorname{RE}(\ell)$, for some $\ell$. Remarkably, the size of the configurations is bounded, unlike those of $\lambda^{[]}$, where histories $\eta$ could grow unbound.

Operational semantics of $\lambda^{\text {check }}$

$$
\begin{gathered}
\frac{\Phi \vdash \sigma, e_{1} \rightarrow \sigma^{\prime}, e_{1}^{\prime}}{\Phi \vdash \sigma, e_{1} e_{2} \rightarrow \sigma^{\prime}, e_{1}^{\prime} e_{2}} \quad \frac{\Phi \vdash \sigma, e_{2} \rightarrow \sigma^{\prime}, e_{2}^{\prime}}{\Phi \vdash \sigma, v e_{2} \rightarrow \sigma^{\prime}, v e_{2}^{\prime}} \\
\Phi \vdash \sigma,\left(\lambda_{z} x . e\right) v \rightarrow \sigma, e\left\{v / x, \lambda_{z} x . e / z\right\} \quad \Phi \vdash \sigma, \text { if } b \text { then } e_{0} \text { else } e_{1} \rightarrow \sigma, e_{\mathcal{B}(b)} \\
\frac{\left\{\varphi\left(c^{\prime}\right) \in \Phi^{\prime} \mid \varphi \in \Phi \wedge \delta\left(\sigma\left(\varphi\left(c^{\prime}\right)\right), \alpha(c)\right)=q_{s}\right\}=\emptyset}{\Phi \vdash \sigma, \text { check } \Phi^{\prime} \text { in } \alpha(c) \rightarrow \delta(\sigma, \alpha(c)), *} \\
\frac{\Phi \cup\{\varphi\} \vdash \sigma, e \rightarrow \sigma^{\prime}, e^{\prime}}{\Phi \vdash \sigma, \text { enter } \varphi \text { in } e \rightarrow \sigma^{\prime}, \text { enter } \varphi \text { in } e^{\prime}} \quad \Phi \vdash \sigma, \text { enter } \varphi \text { in } v \rightarrow \sigma, v
\end{gathered}
$$

The first four rules are straightforward. The rule for an event $\alpha(c)$ guarded by $\Phi^{\prime}$ requires that any policy $\varphi\left(c^{\prime}\right) \in \Phi^{\prime}$ such that $\varphi$ is active, does not lead to the sink state upon $\alpha(c)$. The statement enter $\varphi$ in $e$ is similar to a block in programming languages: the policy $\varphi$ is active while reducing $e$, and its scope is left when $e$ eventually becomes a value.

We are now ready to instrument an expression $e$ in $\lambda^{[]}$and obtain an equivalent expression $\operatorname{instr}_{\mathrm{RE}}(e)$ in $\lambda^{\text {check }}$. In the definition below, we use the risky events collected in RE by the analysis on history grammars. The first rule discards all the policy framings, but records entering the scope of a policy. The other interesting rule is that for instrumenting events: an event $\alpha^{\ell}(c), H$-risky for $\Phi$, becomes guarded by a check on all the policies in $\Phi$. 
Instrumentation of $\lambda^{[]}$expressions

$$
\begin{gathered}
\operatorname{instr}_{\mathrm{RE}}(\varphi[e])=\text { enter } \varphi \text { in } \operatorname{instr}_{\mathrm{RE}}(e) \quad \operatorname{instr}_{\mathrm{RE}}(*)=* \quad \operatorname{instr}_{\mathrm{RE}}(x)=x \\
\operatorname{instr}_{\mathrm{RE}}(c)=c \quad \operatorname{instr}_{\mathrm{RE}}\left(\alpha^{\ell}(r)\right)=\operatorname{check} \mathrm{RE}(\ell) \text { in } \alpha(r) \\
\operatorname{instr}_{\mathrm{RE}}\left(\lambda_{z} x . e\right)=\lambda_{z} x . \operatorname{instr}_{\mathrm{RE}}(e) \quad \operatorname{instr}_{\mathrm{RE}}\left(e_{0} e_{1}\right)=\operatorname{instr}_{\mathrm{RE}}\left(e_{0}\right) \operatorname{instr}_{\mathrm{RE}}\left(e_{1}\right) \\
\operatorname{instr}_{\mathrm{RE}}\left(\text { if } b \text { then } e_{0} \text { else } e_{1}\right)=\text { if } b \text { then } \operatorname{instr}_{\mathrm{RE}}\left(e_{0}\right) \text { else instr } \operatorname{ing}_{\mathrm{RE}}\left(e_{1}\right)
\end{gathered}
$$

The correctness of instrumentation is stated by the following theorem: each execution step of $e$ in $\lambda^{[]}$corresponds exactly to one step of $\operatorname{instr}_{\mathrm{RE}}(e)$ in $\lambda^{\text {check }}$.

Theorem 4. For all histories $\eta$, non-failure expressions $e, e^{\prime}$, and integers $n$ :

$$
\varepsilon, e \rightarrow^{n} \eta, e^{\prime} \Longleftrightarrow \emptyset \vdash \sigma_{0}, \operatorname{instr}_{\mathrm{RE}}(e) \rightarrow^{n} \delta\left(\sigma_{0}, \eta\right), \operatorname{instr}_{\mathrm{RE}}\left(e^{\prime}\right)
$$

\section{Conclusions}

We have proposed a mixed approach to history-based access control. To this aim, we have exploited $\lambda^{[]}$, an extension of the $\lambda$-calculus that allows for security policies with possibly nested, local scopes [4].

We have defined a type and effect system to extract from a given program a history grammar that approximates its run-time behaviour, as far as security is concerned. A history grammar is a context-free grammar that generates execution histories while recording the scope of local policies.

Dynamic checking history-based policies is in general unfeasible, because histories may grow unbound. We have been able to transform programs to record just the abstraction of the history needed to guarantee security, i.e. where and which policies have to be checked. This relies on a (polynomial-time) static analysis on history grammars, which detects the events that are risky, and the policies they may violate. Local checks of risky events can then replace local policies, thus making the dynamic control of accesses feasible.

Colcombet and Fradet [8] and Marriot, Stuckey and Sulzmann [15] mixed static and dynamic techniques to transform programs and make them obey a given global policy. The approach of [8] abstracts a program into a control flow graph, which is then instrumented with some annotations, to track the state of the finite-state automaton that enforces the global property. A minimization phase follows, to remove the unnecessary tracking. Finally, the optimized control flow graph is converted back to a program, that is guaranteed to abort just before violating the property. The approach of [15] is based on over-approximating the run-time behaviour of a program through a context-free grammar. A finite-state automaton models the permitted resource usages. If the language generated by the grammar is not included in the language accepted by the automaton, the 
program is instrumented with the local checks and tracking operations needed to make it obey the policy. Compared to $[8,15]$, our programming model allows for local policies and access events parametrized over resources, while the others only consider global policies and no parametrized events.

Igarashi and Kobayashi proposed in [13] a unified framework for analysing the usage of resources. This is based on an extension of the $\lambda$-calculus that features primitives for creating and accessing resources, and for defining their permitted usage patterns. The resource usage problem requires to approximate the use function that maps expressions to the sequences of possible usage patterns. An execution is resource-safe when the possible patterns are contained in the permitted ones. A type system guarantees that well-typed expressions are resource-safe. Compared to the calculus of [13], $\lambda^{[]}$has no primitive for resource creation, but we plan to introduce it in future work. Indeed, the programming model of [13] is even too powerful: as a result, no complete algorithm exists to verify that inferred usages conform to the permitted ones. Instead, in [4] we provided $\lambda^{[]}$with a static technique to verify when a program is secure.

Skalka and Smith proposed $\lambda_{\text {hist }}$ [19], a $\lambda$-calculus with local security checks that enforce linear $\mu$-calculus properties $[7,14]$ on the past history. A type and effect system approximates the possible run-time histories. Type safety ensures that a typable expression will not go wrong if its effect is valid, i.e. all the histories it represents always pass the local security checks. The validity of effects can be statically verified by model checking $\mu$-calculus formulae over Basic Process Algebras [6,10]. Compared to Skalka and Smith's $\lambda_{\text {hist }}$, our $\lambda^{[]}$features a different programming construct for access control: while in $\lambda_{\text {hist }}$ the access control tests are dictated by local checks inserted into programs, we have policy framings, that localize the time intervals where safety policies must be enforced.

Walker [21] explored an alternative approach to access control, that mixes static and dynamic techniques with proof-carrying code [16]. Security properties are specified by security automata $[5,17]$. When a security-unaware program is compiled, a centralized security policy tells where to insert local checks, in order to obtain provably-secure compiled code. An optimization phase follows: whenever a security check is removed, it is replaced by a proof that the optimized code is still safe. This is done through typed compilation schemata: types encode assertions about program security, ensuring that no run-time violation of the security properties will occur. Before executing a piece of code, a certified verification software ensures that it respects the centralized security policy. Thus, compilers are no longer required to belong to the trusted computing base.

Our previous work [4] has a type and effect system, similar to the present one, whose effects are instead history expressions, equivalent to Basic Process Algebras. These effects are model checked with specially-tailored Büchi automata, to detect whether the program under analysis never goes wrong. In [3] we further refined this model to include liveness properties and call-by-contract service invocation, thus providing a framework for secure service composition. Compared with $[4,3]$, the present paper never rejects programs that possibly go wrong, by mechanically adding the necessary run-time checks only. 
Acknowledgments. We wish to thank the anonymous referees for their insightful comments. Research partially supported by the Project FET-GC II Sensoria.

\section{References}

1. M. Abadi and C. Fournet. Access control based on execution history. In Proc. 10th Annual Network and Distributed System Security Symposium, 2003.

2. A. Banerjee and D. A. Naumann. History-based access control and secure information flow. In Workshop on Construction and Analysis of Safe, Secure and Interoperable Smart Cards (CASSIS), 2004.

3. M. Bartoletti, P. Degano, and G. L. Ferrari. Enforcing secure service composition. In Proc. 18th Computer Security Foundations Workshop (CSFW), 2005.

4. M. Bartoletti, P. Degano, and G. L. Ferrari. History based access control with local policies. In Proc. Fossacs, 2005.

5. L. Bauer, J. Ligatti, and D. Walker. More enforceable security policies. In Foundations of Computer Security (FCS '02), 2002.

6. J. A. Bergstra and J. W. Klop. Algebra of communicating processes with abstraction. Theoretical Computer Science, 37:77-121, 1985.

7. J. C. Bradfield. On the expressivity of the modal mu-calculus. In Proc. International Symposium on Theoretical Aspects of Computer Science, 1996.

8. T. Colcombet and P. Fradet. Enforcing trace properties by program transformation. In Proc. 27th ACM SIGPLAN-SIGACT Symposium on Principles of Programming Languages, 2000.

9. G. Edjlali, A. Acharya, and V. Chaudhary. History-based access control for mobile code. In Secure Internet Programming, 1999.

10. J. Esparza. On the decidability of model checking for several $\mu$-calculi and Petri nets. In Proc. 19th Int. Colloquium on Trees in Algebra and Programming, 1994.

11. P. W. Fong. Access control by tracking shallow execution history. In IEEE Symposium on Security and Privacy, 2004.

12. C. Fournet and A. D. Gordon. Stack inspection: theory and variants. ACM Transactions on Programming Languages and Systems, 25(3):360-399, 2003.

13. A. Igarashi and N. Kobayashi. Resource usage analysis. In Proc. 29th ACM SIGPLAN-SIGACT Symposium on Principles of Programming Languages, 2002.

14. D. Kozen. Results on the propositional $\mu$-calculus. Theoretical Computer Science, 27:333-354, 1983.

15. K. Marriott, P. J. Stuckey, and M. Sulzmann. Resource usage verification. In Proc. First Asian Programming Languages Symposium, 2003.

16. G. C. Necula. Proof-carrying code. In Proc. 24th ACM SIGPLAN-SIGACT Symposium on Principles of Programming Languages, 1997.

17. F. B. Schneider. Enforceable security policies. ACM Transactions on Information and System Security (TISSEC), 3(1):30-50, 2000.

18. P. Sewell and J. Vitek. Secure composition of untrusted code: box- $\pi$, wrappers and causality types. Journal of Computer Security, 11(2), 2003.

19. C. Skalka and S. Smith. History effects and verification. In Asian Programming Languages Symposium, 2004.

20. J.-P. Talpin and P. Jouvelot. The type and effect discipline. In Proc. 7th IEEE Symposium on Logic in Computer Science, 1992.

21. D. Walker. A type system for expressive security policies. In Proc. 27th ACM SIGPLAN-SIGACT Symposium on Principles of Programming Languages, 2000. 